ARTÍCULO

\title{
Importancia del sector forestal en la contabilidad de gases de efecto invernadero (GEI) del país.
}

\author{
Yasna Rojas $^{1 *}$, Carlos Büchner ${ }^{1}$, Marjorie Martin ${ }^{1}$, Sabine Müller-Using ${ }^{1}$ y Carlos Bahamondez ${ }^{1}$ \\ ${ }^{1}$ Instituto Forestal, Sede Los Ríos. yasna.rojas@infor.cl \\ ${ }^{*}$ Autor de correspondencia
}

DOI: https://doi.org/10.52904/0718-4646.2021.558

Recibido: 21.09.2021; Aceptado 17.12.2021

\begin{abstract}
RESUMEN
El sector forestal a través del bosque nativo, las plantaciones y los productos de madera recolectada juega un rol muy importante en el país, contribuye a la mitigación del cambio climático y es el principal sumidero de carbono. En el año 2018 , contribuyó al balance de gases de efecto invernadero disminuyendo en $57 \%$ las emisiones del país, con una absorción neta de $-68.878,3$ kt $\mathrm{CO}_{2}$ eq. Desde 1990 hasta 2018 el sector ha aumentado las absorciones de carbono, en directa relación con el aumento de la superficie de bosques (forestación) y la incorporación de bosque nativo bajo manejo. En el caso del bosque nativo, se observa que la mayor contribución proviene de los bosques de renovales y de las áreas de conservación, mientras que en las plantaciones, la especie Pinus radiata es la que domina con una mayor absorción dada la mayor superficie respecto a otras especies exóticas. Las emisiones de GEI se relacionan directamente con el aumento de las cosechas de trozas y el consumo de leña, mientras que los incendios juegan un rol fundamental en el incremento de emisiones en años puntuales y se observa un aumento de la frecuencia de estos en los últimos años. El sector forestal tiene un potencial para aumentar su capacidad de sumidero de carbono, sin embargo, es necesario considerar todas las variables que afectan las absorciones y las emisiones que afectan al sector.
\end{abstract}

Palabras clave: Cambio climático, bosques, GEI.

\section{SUMMARY}

Through the native forest, plantations and harvested wood products the forestry sector plays a very important role in the country, contributes to the mitigation of climate change and is the main carbon sink. In 2018 , it contributed to the greenhouse gas balance by reducing the country's emissions by $57 \%$, with a net absorption of $-68,878.3 \mathrm{kt} \mathrm{CO} 2 \mathrm{eq}$. From 1990 to 2018, the sector has increased carbon removals, in direct relation to the increase in the area of forests (afforestation) and the incorporation of native forests under management. In the case of the native forest, it is observed that the greatest contribution comes from the second-growth forests and from the conservation areas, while in the plantations, the Pinus radiata species is the one that dominates with a greater absorption given the greater surface area with respect to other exotic species. GHG emissions are directly related to the increase in log harvests and the consumption of firewood, while fires play a fundamental role in the increase of emissions in specific years and an increase in their frequency is observed in recent years. The forestry sector has the potential to increase its carbon sink capacity, however, it is necessary to consider all the variables that affect the removals and emissions that affect the sector.

Keywords: Climatic change, forests, GHG.

\section{INTRODUCCIÓN}

Los bosques naturales primarios y secundarios, así como las plantaciones forestales son importantes sumideros de carbono, fijando el carbono en su biomasa hasta el momento de su cosecha y persistiendo el carbono capturado en la madera durante otro período de tiempo hasta su total degradación. En los ecosistemas forestales, los árboles son el componente más importante de almacenamiento de biomasa y de carbono. El contenido de carbono que se encuentra en los ecosistemas forestales es el resultado de la capacidad de almacenamiento que estos tienen mediante el proceso de fotosíntesis. Las emisiones que se producen en este sector corresponden a las cortas y extracciones de biomasa del bosque y también a eventos que alteran el desarrollo del bosque como los incendios forestales o plagas.

A partir de los acuerdos de la Convención Marco de las Naciones Unidas para el Cambio Climático (CMNUCC) los países llevan la contabilidad de sus emisiones a través de los inventarios de gases de efecto invernadero (GEI). Para ello se consideran en la contabilidad a cinco sectores: (i) Energía; (ii) 
Procesos Industriales y Uso de Productos (IPPU por sus siglas en ingles); (iii) Agricultura; (iv) Uso de la Tierra, Cambio de uso de la tierra y Silvicultura (UTCUTS); y (v) Residuos. En el año 2018, el balance de GEI del país contabilizó $48.321 \mathrm{kt} \mathrm{CO}_{2}$ eq, este balance se produce al totalizar $112.312,6 \mathrm{kt} \mathrm{CO}_{2}$ eq de emisiones y $-63.991,9 \mathrm{kt} \mathrm{CO}_{2}$ eq de absorciones, estas últimas provienen exclusivamente del sector UTCUTS (MMA, 2021).

Por otra parte, el sector UTCUTS considera seis categorías de tierras de acuerdo a las Directrices del IPCC de 2006. (i) tierras forestales; (ii) tierras de cultivo; (iii) pastizales, (iv) humedales; (v) asentamientos, y (vi) otras tierras, además del depósito de carbono en productos de madera. Dentro del sector UTCUTS, las tierras forestales y los productos de madera en el 2018 concentraron una absorción neta de $-61.344,3 \mathrm{kt} \mathrm{CO}_{2}$ eq y $-7.533,7 \mathrm{kt} \mathrm{CO}_{2}$ eq respectivamente, convirtiéndolos en los principales sumideros de carbono del país. Chile cuenta con un importante recurso forestal al año 2018, comprendiendo 16,2 millones de hectáreas de bosque nativo (CONAF para la actualización del inventario de GEI) y 2,3 millones de hectáreas de plantaciones forestales (INFOR, 2019). El presente artículo tiene como objetivo describir el rol que cumple el sector forestal en la contabilidad de gases de efecto invernadero del país e interpretar esta información en el contexto del sector forestal y maderero entre 1990 y 2018.

\section{MATERIAL Y MÉTODO}

En este documento se trabaja con la información proveniente del inventario de gases de efecto invernadero del sector UTCUTS, en conjunto con información estadística del sector, proveniente de los anuarios forestales que publica INFOR. Información que se ha procesado y analizado para facilitar la comprensión del público en general respecto de los distintos elementos que participan en el cálculo de la captura de carbono del sector forestal.

\section{Conceptos}

En la contabilidad de gases de efecto invernadero (GEI) solo se consideran las emisiones y absorciones antrópicas, eso significa que las emisiones y absorciones de gases de efecto invernadero de los inventarios nacionales son el resultado de las actividades humanas.

Se entiende como depósito de carbono, un componente del sistema climático donde el carbono está almacenado. La biomasa forestal, los productos de madera, el suelo y la atmósfera son ejemplos de depósitos de carbono.

Para el sector Agricultura y UTCUTS, las emisiones antrópicas y absorciones por sumideros de gases de efecto invernadero se definen como aquellas que se producen en "tierras gestionadas". La tierra gestionada se define como aquella en la que ha habido intervención humana y donde se han aplicado prácticas para la realización de actividades de producción, ecológicas o sociales (IPCC, 2006). Para el caso de las tierras forestales, el inventario de GEI del país incluye en sus tierras gestionadas a las plantaciones forestales, bosque nativo de segundo crecimiento (renovales), bosque nativo incluido en sistema nacional de áreas silvestres, bosque nativo con plan de manejo según Ley sobre recuperación de bosque nativo y manejo forestal, bosque nativo originado por cambio de cobertura desde plantaciones (restitución), y bosque nativo que se origina de la conversión de otros usos de la tierra (MMA, 2021).

Los bosques de segundo crecimiento que corresponden a renovales y bosque adulto renoval de acuerdo a la clasificación del Catastro de Usos Vegetacionales de CONAF, se mantienen bajo crecimiento hasta alcanzar los $60 \mathrm{~cm}$ de diámetro medio cuadrático (DMC). Una vez que alcanzan este diámetro pasan a ser considerados como bosques adultos en equilibrio, contabilizando un balance neto igual a cero (MMA, 2021).

\section{Determinación del Cambio Anual y Diferencia con Acumulación de Stock}

Para la estimación de los cambios en las existencias de carbono de la biomasa se utiliza el método de pérdidas y ganancias (Ecuación 1), que requiere que la pérdida de carbono de la biomasa se reste de la ganancia de carbono. Las ganancias incluyen el crecimiento de la biomasa en sus componentes aéreos y subterráneos. Las pérdidas están clasificadas en talas o cosechas madereras, recogida y cosecha de leña y pérdidas por incendios o plagas (IPCC, 2006). 


$$
\Delta C_{B}=\Delta C_{G}-\Delta C_{L} \quad \text { Ecuación } 1 \text { (Ecuación } 2.7 \text { IPCC, 2006) }
$$

Donde:

$\Delta C_{B}$ : cambio anual en las existencias de carbono de la biomasa considerando la superficie total (tC/año)

$\Delta C_{G}$ : aumento anual de las existencias de carbono debido al crecimiento de la biomasa para cada categoría de tierra, considerando la superficie total (tC/año)

$\Delta C_{L}$ : reducción anual de las existencias de carbono debida a la pérdida de biomasa para cada categoría de tierra, considerando la superficie total (tC/año)

Las ganancias de las tierras forestales se determinan a partir del crecimiento medio anual por tipo forestal, datos que provienen del Inventario Forestal Nacional (IFN); para el caso de las plantaciones los crecimientos están diferenciados por especie. El volumen es transformado a biomasa, considerando la densidad básica, el factor de expansión a biomasa aérea y la relación entre la biomasa subterránea y aérea según las distintas especies. Toda esta información se encuentra detallada en el Informe Nacional de Inventario de Gases de Efecto invernadero publicado por MMA (2021).

La pérdida anual de biomasa corresponde a la suma de las cosechas de trozas, leña y otras pérdidas producidas por perturbaciones, como los incendios y plagas. En el caso de los incendios, además de estimar las emisiones de carbono, se requiere estimar las emisiones gases no- $\mathrm{CO}_{2}$ como el metano y el óxido nitroso.

\section{RESULTADOS}

Se presenta la información de absorciones y emisiones para bosque nativo, plantaciones, productos de madera y emisiones de incendios, de acuerdo a los resultados publicados en el Informe del Inventario Nacional de GEI (1990-2018) (MMA, 2021). Los resultados se presentan mostrando la evolución desde 1990 y cada diez años hasta el 2018.

\section{Bosque Nativo}

\section{-Absorciones}

El bosque nativo contribuye a la absorción de carbono a través de su crecimiento anual. Los crecimientos de estos bosques fluctúan entre 0,5 a $6,6 \mathrm{~m}^{3} / \mathrm{ha} / \mathrm{año}$ para los distintos tipos forestales de bosque nativo presentes en el inventario de GEI (INFOR, 2016), lo que equivale a capturas anuales de 0,9 a 12,8 $\mathrm{tCO}_{2}$ eq/año en biomasa total.

Al año 2018, la superficie total de bosque nativo que se considera en la contabilidad del inventario de GEI alcanza las 8,03 millones de hectáreas, de las cuales 55,6\% corresponde a bosques de segundo crecimiento y $22,9 \%$ a bosques que crecen en parques y reservas (Cuadro 1). Se destaca la superficie de bosque nativo que ingresa con planes de manejo con una participación del 16,6\%. La superficie de tierras convertidas a bosque nativo, corresponde a superficies de pastizales que son abandonados y luego se regeneran con especies nativas $(4 \%)$. La restitución corresponde a superficie de plantaciones forestales que se convierte a bosque nativo $(0,4 \%)$. Es importante señalar que existe una proporción de superficie de bosque nativo que cada año llega a los $60 \mathrm{~cm}$ de diámetro (DMC), alcanzando el equilibrio definido en el inventario de gases, con lo cual la absorción neta es igual a cero; en el 2018 esta superficie representa el $0,6 \%$ de la superficie de bosque nativo contabilizada en el inventario de gases de efecto invernadero. 
Ciencia \& Investigación Forestal Vol. 27 №3. Diciembre 2021.

Cuadro 1. Participación de bosque nativo según categoría de bosque entre 1990 y 2018

\begin{tabular}{lrrrrr}
\hline \multirow{2}{*}{ Bosque nativo } & \multicolumn{4}{c}{ Superficie (ha) } & Participación \\
\cline { 2 - 6 } & $\mathbf{1 9 9 0}$ & $\mathbf{2 0 0 0}$ & $\mathbf{2 0 1 0}$ & $\mathbf{2 0 1 8}$ & $\begin{array}{c}\text { Porcentual } \\
\text { año 2018 }\end{array}$ \\
\hline Renovales & 4.487 .884 & 4.480 .842 & 4.473 .677 & 4.465 .385 & $55,6 \%$ \\
\hline Áreas de conservación & 1.845 .445 & 1.843 .534 & 1.841 .513 & 1.839 .062 & $22,9 \%$ \\
\hline Bosque nativo Plan de manejo & 157.583 & 909.309 & 1.141 .868 & 1.329 .580 & $16,6 \%$ \\
\hline Restitución & 3.160 & 34.762 & 51.745 & 30.539 & $0,4 \%$ \\
\hline Tierras Convertidas a Bosque Nativo & 21.627 & 237.892 & 437.173 & 324.368 & $4,0 \%$ \\
\hline Bosque nativo en equilibrio & 15.451 & 24.404 & 33.589 & 44.333 & $0,6 \%$ \\
\hline Total & $\mathbf{6 . 5 3 1 . 1 5 0}$ & $\mathbf{7 . 5 3 0 . 7 4 3}$ & $\mathbf{7 . 9 7 9 . 5 6 5}$ & $\mathbf{8 . 0 3 3 . 2 6 7}$ & $\mathbf{1 0 0 \%}$ \\
\hline
\end{tabular}

En el Cuadro 2 se puede ver la evolución de las absorciones de bosque nativo por depósito de carbono. El crecimiento en biomasa total es el que tiene la mayor participación en las absorciones y dentro de éste, se ve que los renovales son los que más contribuyen, alcanzando los mayores aportes entre los años 2000 y 2010. Si bien se observa una disminución en superficie en el mismo período, las mayores absorciones se deben a que los renovales alcanzan mayores crecimientos anuales. Se observa la misma tendencia en las áreas de conservación, solo que los mayores crecimientos se alcanzan entre el 2010 y 2018.

Cuadro 2. Absorciones de bosque nativo para algunos años de la serie temporal del INGEI

\begin{tabular}{lrrrr}
\hline Depósito de carbono & \multicolumn{4}{c}{ Absorciones $\left(\mathbf{k t} \mathbf{C O}_{\mathbf{2}} \mathbf{~ e q}\right)$} \\
\cline { 2 - 5 } & \multicolumn{1}{c}{$\mathbf{1 9 9 0}$} & $\mathbf{2 0 0 0}$ & $\mathbf{2 0 1 0}$ & \multicolumn{1}{c}{$\mathbf{2 0 1 8}$} \\
\hline Biomasa & $\mathbf{- 6 3 . 3 9 8 , 7}$ & $\mathbf{- 7 8 . 2 0 2 , 0}$ & $\mathbf{- 8 1 . 5 6 7 , 1}$ & $\mathbf{- 8 0 . 6 0 3 , 1}$ \\
\hline Renovales & $-43.965,4$ & $-49.248,1$ & $-49.405,6$ & $-47.129,0$ \\
\hline Áreas de conservación & $-18.183,0$ & $-19.923,0$ & $-20.391,8$ & $-20.108,1$ \\
\hline Planes de manejo & $-1.223,1$ & $-8.731,2$ & $-11.237,2$ & $-12.979,7$ \\
\hline Restitución & $-3,5$ & $-38,8$ & $-57,7$ & $-33,9$ \\
\hline Conversión de otros usos & $-23,7$ & $-260,8$ & $-474,8$ & $-352,4$ \\
\hline Materia orgánica muerta & $\mathbf{- 0 , 0 3}$ & $\mathbf{- 0 , 3}$ & $\mathbf{- 0 , 5}$ & $\mathbf{- 1 , 2}$ \\
\hline Carbono del suelo & $\mathbf{- 2 , 6}$ & $\mathbf{- 2 8 , 6}$ & $\mathbf{- 4 0 , 9}$ & $\mathbf{- 2 2 , 6}$ \\
\hline Total & $\mathbf{- 6 3 . 4 0 1 , 4}$ & $\mathbf{- 7 8 . 2 3 1 , 0}$ & $\mathbf{- 8 1 . 6 0 8 , 5}$ & $\mathbf{- 8 0 . 6 2 6 , 9}$ \\
\hline
\end{tabular}

(Fuente: Elaboración propia a partir de INGEI 1990-2018)

En el caso del bosque nativo manejado, las absorciones aumentan ya que cada año se incorpora nueva superficie manejada. La restitución y la conversión de otros usos tienen las menores absorciones debido a que la superficie es menor y además el bosque nativo que aparece en estos terrenos corresponde a matorral arborescente con un crecimiento anual muy bajo comparado al crecimiento de los bosques que están en las otras categorías.

Los depósitos de carbono de materia orgánica muerta y carbono del suelo también son valores menores ya que se originan por la restitución y conversión de otros usos, generando mejoras en el carbono del suelo, pero debido a que las superficies anuales son muy marginales, la participación de estos depósitos de carbono también resulta marginal.

\section{-Emisiones}

Las emisiones que afectan al bosque nativo se deben principalmente al consumo de leña y a la cosecha de trozas. En el caso de la cosecha de trozas, la producción ha venido disminuyendo desde 1990, cuando alcanzó los 2,3 millones de metros cúbicos, hasta el 2018 en que solo llega a los 260 mil metros cúbicos. La participación de especies nativas en el consumo industrial de madera en troza ha descendido a menos del 2\% en los últimos años (INFOR, 2019; Martin et al., 2020).

Por otra parte, el consumo de leña de especies nativas ha sido muy importante (Cuadro 3), alcanzando un máximo de emisiones entre el 2008 y 2009 con valores de $12.900 \mathrm{kt}$ de $\mathrm{CO}_{2}$ eq, con 9,2 millones de metros cúbicos de consumo de leña (63\% del consumo nacional). A partir del 2010 se observa una disminución de estas emisiones que llegan a las $8.240 \mathrm{kt}$ de $\mathrm{CO}_{2}$ eq en el 2018, siendo responsables del $46 \%$ del consumo nacional (5,8 millones de metros cúbicos). La disminución del consumo de leña de bosque nativo se ha transformado en un aumento del consumo de leña de especies exóticas. 
Por último, las emisiones por conversión de otros usos corresponden a las emisiones que se producen en el momento de la conversión de tierras que no forestales a bosque nativo. Estas tienen una participación muy marginal en el total de emisiones del sector, ya que la biomasa de estos otros usos de la tierra que se pierde y emite al momento de la conversión es marginal.

Cuadro 3. Emisiones de GEI en bosque nativo

\begin{tabular}{lrrrr}
\hline \multirow{2}{*}{ Fuente } & \multicolumn{4}{c}{ Emisiones $\left.\mathbf{( k t} \mathbf{C O}_{2} \mathbf{~ e q}\right)$} \\
\cline { 2 - 5 } & \multicolumn{1}{c}{$\mathbf{1 9 9 0}$} & $\mathbf{2 0 0 0}$ & $\mathbf{2 0 1 0}$ & $\mathbf{2 0 1 8}$ \\
\hline Cosecha trozas & $4.408,0$ & $2.842,9$ & 817,6 & 501,2 \\
\hline Consumo de leña & $6.731,2$ & $10.764,1$ & $11.229,1$ & $8.240,1$ \\
\hline Conversión de otros usos & 302,0 & 302,0 & 243,5 & 67,7 \\
\hline Total & $\mathbf{1 1 . 4 4 1 , 2}$ & $\mathbf{1 3 . 9 0 8 , 9}$ & $\mathbf{1 2 . 2 9 0 , 2}$ & $\mathbf{8 . 8 0 9 , 0}$ \\
\hline
\end{tabular}

\section{Plantaciones}

\section{-Absorciones}

En el caso de las plantaciones, los crecimientos en volumen fustal varían entre 2,3 y 41,0 m³/ha/año, los crecimientos más bajos corresponden a especies como tamarugo y algarrobo y los mayores crecimientos a Eucalyptus nitens. Al año 2018 se cuenta con una superficie de plantaciones de 2,28 millones de hectáreas; la distribución de superficie de plantaciones por especie (Cuadro 4) muestra que el 56\% corresponde a Pinus radiata, mientras que Eucalyptus sp. concentra un $38 \%$ de la superficie, el resto de las especies tienen una participación menor al $5 \%$ de la superficie total de plantaciones. Es importante destacar que dentro de la categoría Otras especies, existe participación de especies nativas, que al año 2018 alcanzó el 0,29\% de la superficie total de plantaciones.

Cuadro 4. Distribución de superficie de plantación por especies

\begin{tabular}{lrrrrr}
\hline \multicolumn{1}{c}{ Especie } & \multicolumn{4}{c}{ Superficie (ha) } & $\begin{array}{r}\text { Participación } \\
\text { porcentual } \\
\text { año 2018 (\%) }\end{array}$ \\
\cline { 2 - 6 } & \multicolumn{1}{c}{$\mathbf{1 9 9 0}$} & $\mathbf{2 0 0 0}$ & $\mathbf{2 0 1 0}$ & $\mathbf{2 0 1 8}$ & $56 \%$ \\
\hline Pinus radiata & 1.243 .293 & 1.474 .773 & 1.471 .806 & 1.285 .640 & $26 \%$ \\
\hline Eucaliptus globulus & 101.700 & 256.031 & 500.428 & 585.272 & $12 \%$ \\
\hline Eucaliptus nitens & - & 102.585 & 196.966 & 273.043 & $1 \%$ \\
\hline Prosopis chilensis y P. tamarugo & 23.801 & 24.165 & 25.873 & 26.322 & $1 \%$ \\
\hline Pseudotsuga menziesii & 11.343 & 14.286 & 16.867 & 16.507 & $0 \%$ \\
\hline Populus spp. & 3.526 & 4.415 & 6.097 & 5.049 & $4 \%$ \\
\hline Otras especies & 38.989 & 60.216 & 93.419 & 85.607 & $\mathbf{1 0 0 \%}$ \\
\hline Total & $\mathbf{1 . 4 2 2 . 6 5 2}$ & $\mathbf{1 . 9 3 6 . 2 0 7}$ & $\mathbf{2 . 3 1 1 . 4 5 5}$ & $\mathbf{2 . 2 7 9 . 4 5 8}$ & \\
\hline & \multicolumn{2}{c}{${ }^{*}$ Incluye especies nativas } & &
\end{tabular}

Si se analiza la información respecto a las absorciones (Cuadro 5), en el año 2018 Pinus radiata contribuyó con $67 \%$ de las absorciones de $\mathrm{CO}_{2}$ de la biomasa total (biomasa aérea y radicular), le sigue Eucalyptus spp. con un aporte de $29 \%$ de las absorciones. El resto de las especies tiene un aporte menor al $3 \%$. Si bien pino radiata tiene crecimientos menores que eucalipto, aporta en forma importante a las absorciones debido a la gran superficie presente en el país.

Cuadro 5. Absorciones de GEI en plantaciones por depósito de carbono y especie

\begin{tabular}{lrrrr}
\hline \multirow{2}{*}{ Depósito de carbono } & \multicolumn{4}{c}{ Absorciones $\left(\mathbf{k t ~ C O}_{\mathbf{2}} \mathbf{~ e q}\right)$} \\
\cline { 2 - 5 } & $\mathbf{1 9 9 0}$ & $\mathbf{2 0 0 0}$ & $\mathbf{2 0 1 0}$ & $\mathbf{2 0 1 8}$ \\
\hline Crecimiento de la biomasa & $\mathbf{- 3 6 . 7 6 8 , 6}$ & $\mathbf{- 5 5 . 1 0 6 , 8}$ & $\mathbf{- 7 2 . 1 0 9 , 3}$ & $\mathbf{- 7 5 . 1 2 0 , 6}$ \\
\hline Pinus radiata & $-31.624,5$ & $-37.499,1$ & $-37.396,0$ & $-32.699,8$ \\
\hline Eucalyptus globulus & $-3.787,7$ & $-9.591,8$ & $-20.287,9$ & $-23.856,7$ \\
\hline Eucalyptus nitens & & $-6.131,9$ & $-11.706,0$ & $-16.096,9$ \\
\hline Otras especies & $-1.356,4$ & $-1.884,0$ & $-2.719,3$ & $-2.467,2$ \\
\hline Carbono del suelo & $\mathbf{- 8 6 5 , 3}$ & $\mathbf{- 6 1 1 , 4}$ & $\mathbf{- 4 8 5 , 9}$ & $\mathbf{- 4 2 2 , 0}$ \\
\hline Total & $\mathbf{- 3 7 . 6 3 3 , 9}$ & $\mathbf{- 5 5 . 7 1 8 , 2}$ & $\mathbf{- 7 2 . 5 9 5 , 2}$ & $\mathbf{- 7 5 . 5 4 2 , 6}$ \\
\hline
\end{tabular}


En cuanto a la evolución de las absorciones de carbono de las plantaciones, la especie Pinus radiata predomina con la mayor participación desde 1990, y a medida que aumenta la superficie aumenta la absorción de carbono, alcanzando un máximo de absorción en 2002 con -38.480 kt $\mathrm{CO}_{2}$ eq y 1,51 millones de hectáreas. Luego la superficie se estabiliza en torno a 1,4 millones de hectáreas y $-37.000 \mathrm{kt}$ $\mathrm{CO}_{2}$ eq, y desde 2017 presenta una disminución importante, llegando al 2018 a $-32.699,8$ kt $\mathrm{CO}_{2}$ eq y 1,28 millones de hectáreas. Este decrecimiento se debe tanto al término del programa de incentivo a la forestación y su prórroga (2012), como a la gran pérdida por incendios en el año 2017. Por otra parte, se observa como Eucalyptus globulus y nitens han aumentado en forma sostenida su participación en el tiempo, alcanzando en 2018 una superficie de 858.315 ha y $-39.953,6$ kt $\mathrm{CO}_{2}$ eq de absorciones. Este aumento se debe a diversos factores, donde destacan la preferencia por parte de pequeños y medianos propietarios por estas especies, menor edad de rotación respecto a pino radiata, mayores crecimientos y optimización de uso de suelos en sectores donde pino radiata presenta crecimientos inferiores (Büchner et al., 2018).

También es importante destacar que la superficie de forestación ha disminuido drásticamente en el último decenio (Figura 1), si bien se alcanzaron valores promedio de 60.000 ha anuales entre 1990 y 2007, alcanzando máximos de 70.000 ha, desde el 2008 han descendido desde 30.000 ha anuales hasta llegar al 2018 a 1.425 ha de forestación (INFOR, 2011, INFOR 2020). Debido a que la superficie de plantaciones se mantiene estable, la absorción de carbono también tiende a estabilizarse y así, sin incorporar nuevas áreas plantadas, no hay mayor margen para obtener mayores tasas de absorción, manteniendo un promedio de absorción de -75.000 kt $\mathrm{CO}_{2}$ eq.

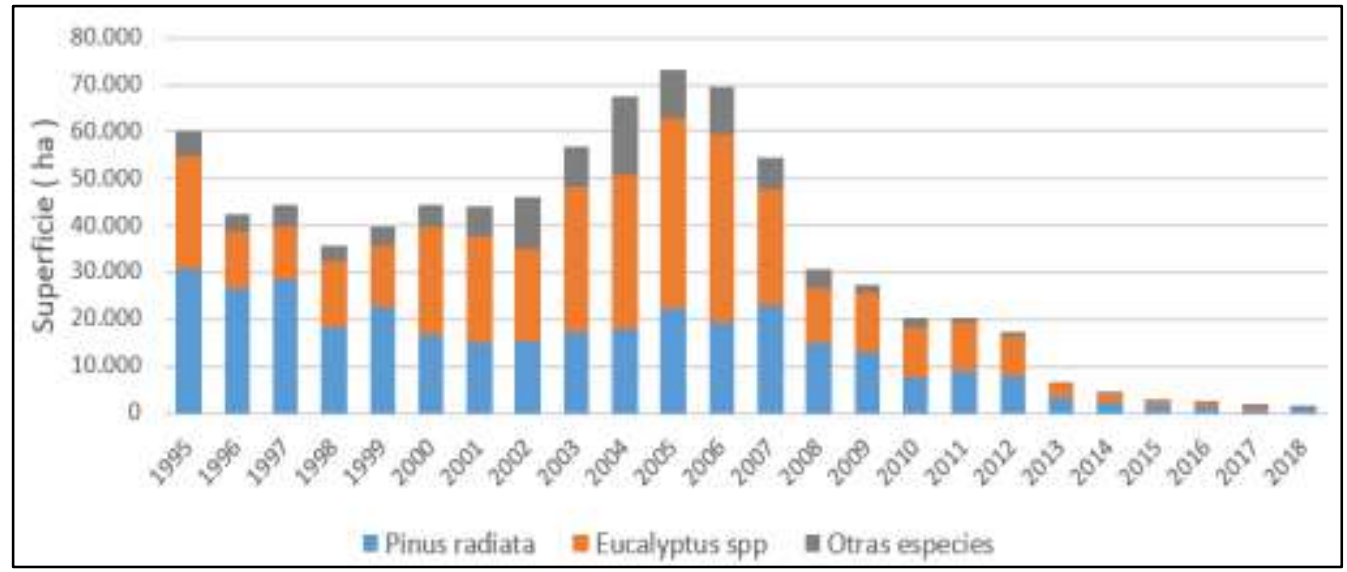

(Fuente: INFOR, 2020a)

Figura 1. Superficie de forestación de plantaciones desde 1995 a 2018

\section{-Emisiones}

En cuanto a las emisiones (Cuadro 6), las mayores emisiones de plantaciones provienen de la cosecha de trozas para fines industriales con $70.542,1 \mathrm{kt} \mathrm{CO}_{2}$ eq en 2018, donde los principales destinos de abastecimiento corresponden al segmento industrial de pulpa y papel, madera aserrada, tableros y chapas, postes y polines, y otros productos (INFOR, 2020a). La industria asociada del sector ha basado su producción y crecimiento en el consumo de madera proveniente de plantaciones de Pinus radiata y Eucaliptos. Actualmente el sector presenta un consumo promedio de 45,4 millones de $\mathrm{m}^{3} \mathrm{ssc}$ de madera en troza (período 2015 - 2019), donde el 98,5\% de este consumo es explicado por estas especies. Una parte de la cosecha industrial se destina a productos de madera aserrada y tableros que se aportan a otro depósito de carbono, los productos de madera, donde queda almacenado el carbono por un período de tiempo. 
Cuadro 6. Emisiones asociadas a plantaciones

\begin{tabular}{lrrrr}
\hline \multicolumn{1}{c}{ Fuente } & \multicolumn{4}{c}{ Emisiones $\left.\mathbf{( k t} \mathbf{C O}_{\mathbf{2}} \mathbf{e q}\right)$} \\
\cline { 2 - 5 } & $\mathbf{1 9 9 0}$ & $\mathbf{2 0 0 0}$ & $\mathbf{2 0 1 0}$ & \multicolumn{1}{c}{$\mathbf{2 0 1 8}$} \\
\hline Cosecha trozas & $\mathbf{1 6 . 6 4 6 , 7}$ & $\mathbf{3 3 . 0 3 9 , 7}$ & $\mathbf{5 2 . 2 6 8 , 0}$ & $\mathbf{7 0 . 5 4 2 , 1}$ \\
\hline${ }^{*}$ Consumo de leña exóticas & $4.363,6$ & $6.977,9$ & $7.279,4$ & $10.759,6$ \\
\hline Conversión de otros usos & $3.909,1$ & $3.907,4$ & $2.205,4$ & $1.074,1$ \\
\hline Materia orgánica muerta & $1.531,7$ & $1.531,7$ & 817,0 & 502,4 \\
\hline Carbono del suelo & 280,0 & 238,5 & 190,0 & 123,8 \\
\hline Total & $\mathbf{2 6 . 7 3 1 , 1}$ & $\mathbf{4 5 . 6 9 5 , 4}$ & $\mathbf{6 2 . 7 5 9 , 7}$ & $\mathbf{8 3 . 0 0 1 , 9}$ \\
\hline * Incluye también especies exóticas que se encuentran en cortinas de árboles y frutales
\end{tabular}

El consumo de leña es el segundo mayor emisor, sin embargo, debe analizarse con cuidado ya que incluye además de especies exóticas de plantaciones forestales, a especies que se encuentran creciendo como cortinas cortavientos o árboles aislados, y a residuos de podas de frutales. Por lo que, las emisiones de leña no pueden atribuirse en su totalidad a las plantaciones forestales, existiendo una fracción que corresponde a la categoría de tierras de pastizales para el caso de árboles que no constituyen bosques, y otra parte a tierras de cultivo para el caso de frutales. No obstante, debido a que la información base de consumo de leña aun no es posible de separar en estas fuentes de origen, todo el consumo de leña de especies exóticas se contabiliza en tierras forestales. Respecto a la tendencia del consumo de leña de especies exóticas, se ve un aumento constante desde 1990 con emisiones de 4.463 kt de $\mathrm{CO}_{2}$ eq hasta el 2018 con casi $11.000 \mathrm{kt} \mathrm{CO}$ eq, correspondientes a un consumo de 6,9 millones de $\mathrm{m}^{3}$, que representan el $54 \%$ del consumo nacional.

Las emisiones por conversión de otros usos a plantaciones forestales también corresponden a la biomasa de otros usos de la tierra, que se considera emitida al momento del cambio de uso de la tierra. Para el caso de las plantaciones, la mayor cantidad de superficie proviene de tierras de pastizales que se han transformado en plantaciones forestales. Y la disminución de estas emisiones en los últimos años tiene relación directa con la menor tasa de forestación y por ende de conversión de otros usos a plantaciones forestales.

Las emisiones por materia orgánica muerta corresponden a las pérdidas de biomasa muerta que se producen al momento de la sustitución de bosque nativo por plantaciones. Las emisiones han ido disminuyendo debido a que la superficie de bosque nativo que se convierte a plantaciones también ha disminuido, sin embargo, aún persiste superficie de bosque nativo que es sustituida por plantaciones.

\section{Productos de madera recolectada}

Los productos de madera recolectada representan un almacenamiento de carbono, ya que parte del carbono de la cosecha se traslada a los productos de madera, los que a su vez van liberando lentamente el carbono a la atmósfera. Las Directrices del IPCC de 2006 establecen que los productos de madera aserrada y tableros pueden almacenar carbono por 30 años y que, para el caso de productos de papel, este puede permanecer por dos años.

La figura 2 muestra la contribución de los productos de madera del sector forestal en Chile y que tiene directa relación con la producción de madera aserrada y tableros (Figura 3) que han tenido una tasa creciente de producción, que alcanzó un máximo en año 2006. Luego hay un descenso en la producción de madera aserrada, siendo el año 2009 cuando se alcanza la menor producción, debido a la crisis financiera mundial, ocasión en que el consumo industrial de trozas en Chile fue 8,7\% menor al año 2008; en particular el rubro más afectado fue el de la producción de madera aserrada, que disminuyó en $20,1 \%$ respecto a la producción del 2008 (INFOR, 2010). Esto afecta directamente al depósito de productos de madera reduciendo su contribución como depósito de carbono. Con posterioridad al 2009 ha habido una recuperación de la producción que muestra su efecto en la absorción de $\mathrm{CO}_{2}$. Sin embargo, el año 2017 también se produce una baja en la producción, debido a los mega incendios que se registraron ese año, donde se vieron afectadas las plantas de aserrío, principalmente de la región del Maule, con el consiguiente impacto en la oferta de madera de plantaciones. La disminución de la producción de madera aserrada alcanzó un 3,6\% respecto al año 2016 y se atribuye a los incendios (INFOR, 2018). 


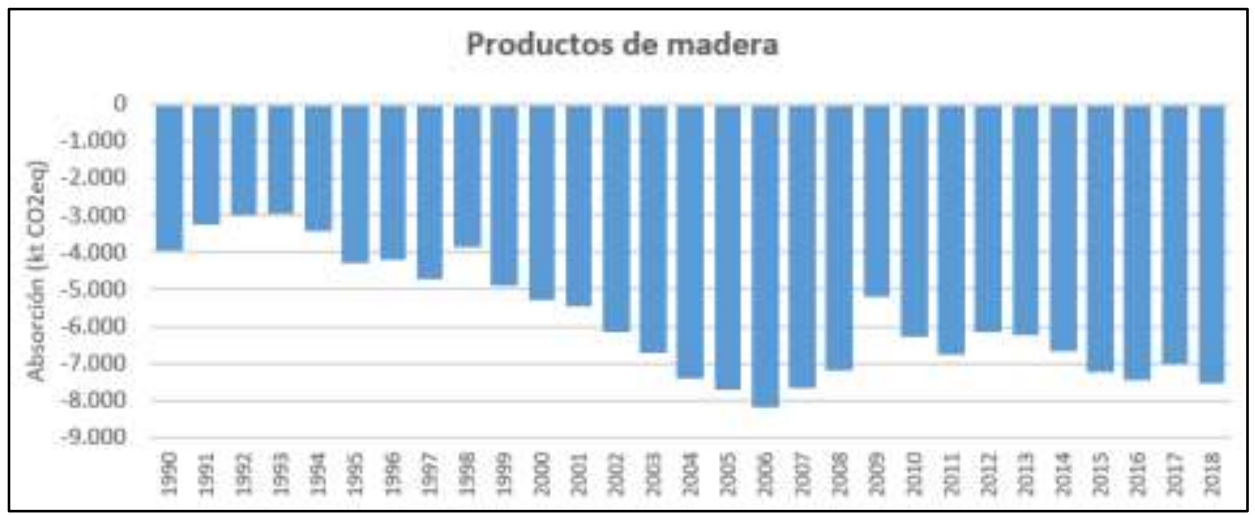

(Fuente: MMA, 2021)

Figura 2. Contribución de productos de madera a la absorción de carbono

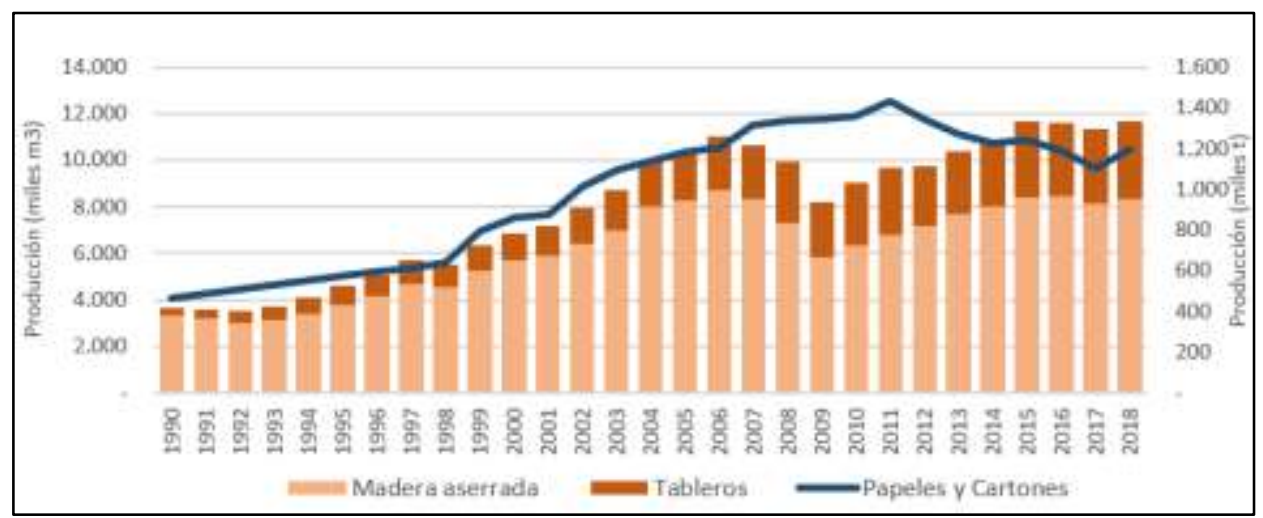

(Fuente: INFOR, 2020a)

Figura 3. Producción nacional de madera aserrada, tableros y papeles y cartones.

Respecto a la producción de madera aserrada (Figura 4) como contribución de depósito de carbono, son las especies exóticas las que aportan en forma mayoritaria. Las bajas tasas de cosecha de trozas de especies nativas dan cuenta de la poca participación de estas especies en este depósito de carbono.

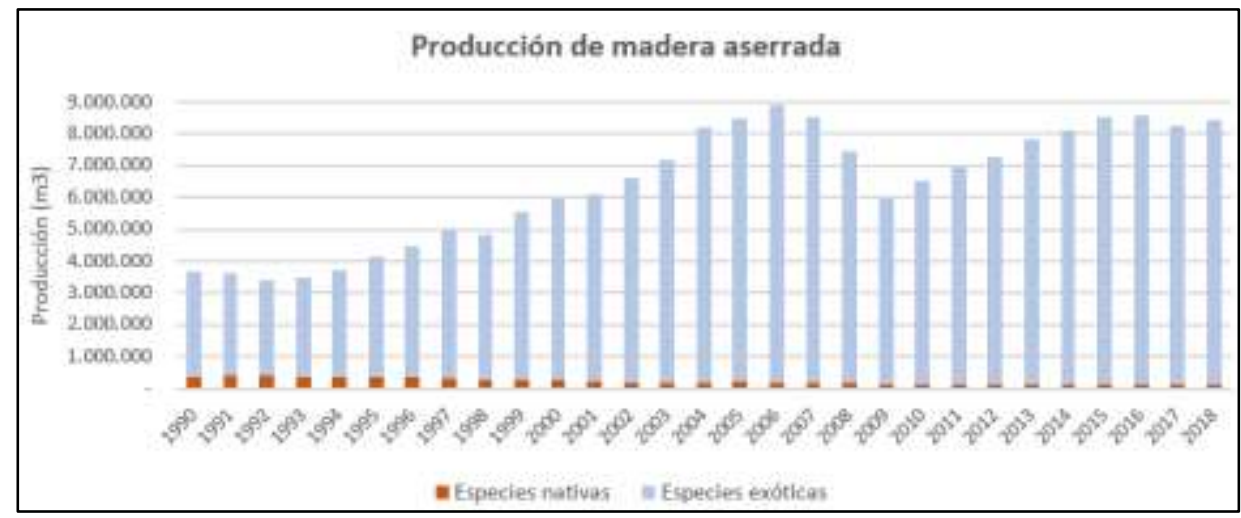

(Fuente: INFOR, 2020a)

Figura 4. Producción de madera aserrada por especies. 


\section{Incendios}

Los incendios forestales influyen en las emisiones del sector forestal porque al incendiarse los bosques, una gran cantidad de biomasa se pierde y emite, en el año en que ocurre el incendio. Estas pérdidas de biomasa se contabilizan como pérdidas de carbono y emisiones de gases no-CO2, como metano y óxido nitroso que se emiten producto de la combustión. La importancia de los incendios forestales radica en que son capaces de generar grandes emisiones y modificar la contribución anual de los bosques a la contabilidad del inventario de GEI.

La Figura 5 muestra el impacto de los incendios en la serie histórica del inventario de GEI, donde se destacan los incendios de 1998, con una superficie de 60.000 ha de bosque nativo que afectó bosques con gran acumulación de biomasa. Ese año el $96 \%$ de la superficie incendiada se concentró en las regiones de Los Ríos y Los Lagos, que de acuerdo al IFN, acumulan un stock de 270 a 328 t/ha de biomasa aérea. Los megaincendios de 2017 afectaron una gran cantidad de superficie, que alcanzó las 281.494 ha de plantaciones y 90.258 ha de bosque nativo, generando emisiones iguales a $57.000 \mathrm{kt} \mathrm{CO}_{2}$ eq, y disminuyendo en un $85 \%$ la absorción neta promedio anual que han tenido las tierras forestales desde 1990 hasta 2016.

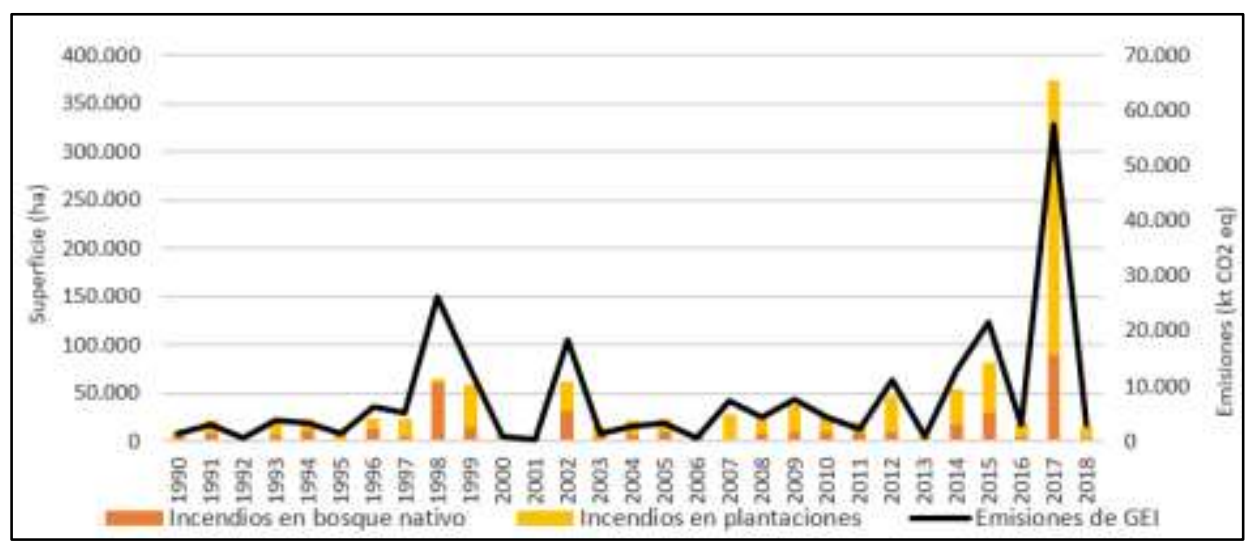

(Fuente: MMA,2021)

Figura 5. Superficie de incendios y emisiones de gases de efecto invernadero, período 1990-2018.

\section{Pérdidas de bosques por conversión a otros usos de la tierra}

El cambio de uso de la tierra desde bosques a otros usos se conoce como deforestación y en la contabilidad del inventario de GEI, estas pérdidas son emisiones que se atribuyen al uso de tierra al cual es convertido el bosque. En el Cuadro 7 se muestran las emisiones producto de las conversiones de bosque nativo -renovales, bosque adulto y matorral arborescente que forma parte del bosque nativo - y bosque mixto y la superficie promedio anual de conversión a los distintos usos de la tierra para tres períodos del inventario de GEI. Cuando se reemplaza un bosque por otro uso de la tierra, se pierde todo el stock de biomasa presente en el terreno junto con la materia orgánica muerta y pérdidas de carbono del suelo. Los valores de biomasa aérea para renovales y bosque adulto pueden variar entre 9 a 310 t/ha dependiendo de la región del país (MMA, 2021 e INFOR, 2016), y en el caso de la materia orgánica muerta, la biomasa varía desde 36 t/ha hasta 166 t/ha (INFOR, 2016).

En la década del 90 se llegaron a perder casi 17.000 ha anuales de bosques para ser convertidas en otros usos de la tierra, los pastizales concentran las mayores tasas de conversión equivalentes al $88 \%$ del total de emisiones y le siguen, muy por debajo, las tierras de cultivo. En la línea temporal se observa una disminución de las conversiones de bosques a pastizales, disminuyendo a casi un $50 \%$ en el período 2010-2018 respecto al período inicial, sin embargo, las tierras forestales convertidas a tierras de cultivo y a asentamientos presentan aumentos de 200 y $300 \%$ respectivamente. Aproximadamente el $50 \%$ de los bosques convertidos a otros usos corresponde a matorral arborescente. 
Ciencia \& Investigación Forestal Vol. 27 №3. Diciembre 2021.

Cuadro 7. Emisiones y superficie anual de tierras forestales convertidas a otros usos de la tierra.

\begin{tabular}{|c|c|c|c|c|c|c|}
\hline \multirow{2}{*}{$\begin{array}{l}\text { Período } \\
\text { Tipo de conversión }\end{array}$} & \multicolumn{2}{|c|}{ 1990-1999 } & \multicolumn{2}{|c|}{$2000-2009$} & \multicolumn{2}{|c|}{$2010-2018$} \\
\hline & $\begin{array}{c}\text { Emisiones } \\
\text { anuales } \\
\text { (kt } \mathrm{CO}_{2} \text { eq) }\end{array}$ & $\begin{array}{l}\text { Superficie } \\
\text { anual } \\
\text { (ha) }\end{array}$ & $\begin{array}{c}\text { Emisiones } \\
\text { anuales } \\
\left(\mathrm{kt} \mathrm{CO}_{2} \text { eq) }\right.\end{array}$ & $\begin{array}{c}\text { Superficie } \\
\text { anual } \\
\text { (ha) }\end{array}$ & $\begin{array}{c}\text { Emisiones } \\
\text { anuales } \\
\text { (kt } \mathrm{CO}_{2} \text { eq) }\end{array}$ & $\begin{array}{c}\text { Superficie } \\
\text { anual } \\
\text { (ha) }\end{array}$ \\
\hline TF a Cultivo & 244 & 1.332 & 395 & 2.170 & 427 & 2.684 \\
\hline TF a Pastizales & 4.010 & 14.793 & 2.869 & 9.759 & 2.505 & 7.963 \\
\hline TF a Humedales & 65 & 165 & 49 & 131 & 15 & 54 \\
\hline TF a Asentamientos & 32 & 316 & 64 & 458 & 98 & 459 \\
\hline TF a Otras tierras & 173 & 233 & 326 & 351 & 378 & 413 \\
\hline Total & 4.523 & 16.839 & 3.703 & 12.870 & 3.423 & 11.573 \\
\hline
\end{tabular}

\section{DISCUSIÓN}

Los bosques en nuestro país actúan como importantes sumideros de carbono para contribuir a la mitigación del cambio climático, ya que las ganancias anuales de carbono son superiores a las emisiones del mismo período. Además, Chile actualizó su Contribución Determinada a nivel nacional (NDC) en el marco del Acuerdo de Paris, aumentando las metas de mitigación en el sector forestal, con 200 mil hectáreas de manejo y recuperación de bosque nativo y 200 mil ha de forestación (Gobierno de Chile, 2020). En este contexto, es importante considerar los factores que influyen en las absorciones y emisiones del sector forestal para mantener o aumentar la condición de sumidero de carbono del país.

En el caso del bosque nativo y la participación de los productos madereros, estos han perdido cada vez más relevancia dentro de los diferentes segmentos industriales y usos finales a nivel nacional. Prueba de esto, las cifras de consumo de madera en troza de especies nativas han descendido a menos del $1 \%$ de participación en los últimos años, presentando un consumo de $224.038 \mathrm{~m}^{3} \mathrm{ssc}$ de volumen de madera en troza al año 2019, principalmente explicado por el sector del aserrío y en menor medida el sector de tableros - chapas (INFOR, 2020a; INFOR, 2020b). Dentro de los múltiples factores asociados a la disminución en el consumo y baja demanda de productos del bosque nativo se encuentran, la calidad maderera del recurso, incertidumbre de abastecimiento de las unidades productivas, aspectos legales (planes de manejo), accesibilidad al recurso, escasa agregación de valor e innovación en los productos, aspectos productivos y de secado, factores que impactan en la cadena logística, costos, como en la calidad final de los productos.

Una segunda dimensión que se asocia a la pérdida del mercado de las maderas nativas, se relaciona con la entrada de otros materiales sustitutos que la reemplazan en el segmento de la construcción, mercados de apariencia, desarrollo de muebles y decoración, entre otros segmentos que son dominados por materiales como el metal, plásticos, tableros de partículas, fibrocemento y otros (INFOR, 2020b).

El estudio de disponibilidad de renovales estima solo para renovales de Roble-Raulí-Coihue un potencial de 702.142 ha de bosques que cumplirían las condiciones necesarias para un manejo con fines productivos (Martin et al. 2020). De este conjunto, hoy solo se manejan 56.842 ha con plan de manejo aprobado por CONAF (Müller-Using et al., 2020).

A pesar de que el inventario de GEl ya tiene contabilizada toda la superficie de renovales con un crecimiento promedio que entrega el IFN, estos bosques podrían aumentar su impacto siempre y cuando se logre mejorar su tasa de crecimiento a través del manejo. Según las tasas de crecimiento de bosques de roble manejados con fines madereros, publicadas recientemente por Pilquinao et al. (2020), la reacción de los árboles a la intervención silvícola significó un $14 \%$ de aumento en el crecimiento promedio de los rodales. Aunque las cifras publicadas muestran el efecto del manejo, también transparentan que a partir de las actuales prácticas silvícolas no se logrará superar el incremento periódico anual que aplica el inventario de GEI. Sin embargo, con un cambio de silvicultura se podría lograr, pues variados estudios han demostrado que un manejo temprano e intensivo de estas formaciones puede duplicar el crecimiento volumétrico anual, respecto de los datos ocupados actualmente en el inventario de GEI (Grosse, 2009; Donoso et al., 1993; Müller-Using et al. 2020).

Otro efecto del manejo silvícola sostenible y dirigido a la producción de madera de alto valor sería aumentar el ciclo de vida de los productos madereros. Actualmente, la leña es el principal producto que se genera del bosque nativo, alcanzando el $46 \%$ del consumo total de leña y un $0,5 \%$ del consumo total de trozas industriales que se destinan a productos aserrables (INFOR, 2020b). Sin embargo, las 
proyecciones de Martin et al. (2020) indican que sería posible aumentar la proporción de madera aserrada, en la gama de productos del bosque nativo, del actual $4 \%$ a un $45 \%$. El supuesto de esta cifra es la incorporación continua de nueva superficie al manejo forestal sustentable y un trozado optimizado que prioriza siempre el producto de mayor valor. De esta manera se podría mejorar considerablemente la contribución de las especies nativas al depósito de carbono de los productos de madera recolectada.

Para lograr cada una de las dos opciones se requiere una revisión profunda de la política forestal del país y sus instrumentos legales, como es la Ley 20.283 de Recuperación del Bosque Nativo y Fomento Forestal, la cual a 13 años de su promulgación no ha mostrado los impactos esperados (Müller-Using, 2020; SIMEF, 2020)

En el caso de las plantaciones forestales, principal fuente de abastecimiento de madera en troza de la industria forestal, se observa un nulo crecimiento respecto a la incorporación de nuevas superficies de plantaciones a través de la forestación, presentando una estabilización respecto a la superficie total plantada en los últimos años. Considerando el período 2010-2019 la superficie de plantaciones se presentó en rangos entre los 2,28 y 2,44 millones de hectáreas, variaciones asociadas a actividades de cosecha, incendios forestales y reforestación de estas áreas.

Respecto a los actuales niveles de producción del sector y el recurso plantaciones, el último estudio de disponibilidad de madera en plantaciones de pino radiata y eucaliptos, presentó para el escenario base una disminución de la proyección de la oferta de volumen de madera disponible (Büchner et al., 2018). Las plantaciones de Eucaliptos presentaron una oferta bastante ajustada respecto a las proyecciones de demanda del sector, esto considerando la proyección de consumo de nuevos proyectos (nueva planta), que, a la fecha, aún no opera a plena capacidad. En cuanto a la oferta de madera en trozas de Pinus radiata, de acuerdo al consumo proyectado, éste se presentaba en déficit, según los efectos estimados de los mega incendios del 2017, proyectando una recuperación en un horizonte cercano a los 20 años posterior a los incendios. El mismo estudio proyecta un escenario de mejoramiento genético en el crecimiento y rendimiento y solo se observaron incrementos para la especie Eucalyptus nitens, ya que la especie Eucalyptus globulus se ve fuertemente afectado por los efectos de la mega sequía y la presencia del defoliador Gonipterus platensis (Coleoptera: Curculionidae) que se alimenta de las hojas y brotes nuevos, afectando el desarrollo de las plantaciones.

Si esta tendencia se mantiene en las plantaciones forestales, sin nuevas forestaciones las absorciones anuales de carbono se mantendrían relativamente constantes en el tiempo, las emisiones por cosecha aumentarían en la medida que los rendimientos de las plantaciones lo permitan. Para el caso de los productos de madera hay oportunidades de crecer como depósito de carbono, siempre que la industria destine la producción a productos de madera aserrada y tableros

Las dos variables que afectan a los bosques como sumideros de carbono son la deforestación y los incendios forestales. Al analizar el INGEI se ve que las tasas de deforestación han disminuido desde 1990 a la fecha, pero siguen existiendo con tasas anuales importantes e impactan no solo por la pérdida de carbono, sino por la pérdida de biodiversidad, producto de la fragmentación del paisaje (Echeverría et al. 2006).

Por otra parte, los incendios forestales afectan de manera importante la contribución anual de los bosques como sumidero de carbono. En los últimos años en Chile, estos eventos han afectado a grandes superficies de plantaciones y vegetación natural. Al analizar las cifras de incendios para el período 20102018, se observa un aumento de incendios de gran magnitud, considerando los años 2014, 2015 y los mega incendios del 2017 (CONAF, 2021). Esta tendencia se explica principalmente por factores asociados al cambio climático, considerando la prolongada y extensa sequía en la zona centro-sur del país, olas de calor, y la actividad humana (González et al. 2020). Por lo tanto, es importante, centrar los esfuerzos en disminuir estas emisiones.

\section{CONCLUSIONES}

Las tierras forestales son los principales sumideros de carbono del país y junto a los productos de madera concentraron en el 2018 una absorción neta de $-67.878,0$ kt $\mathrm{CO}_{2}$ eq. Si bien las absorciones de carbono provenientes de plantaciones son solo un $6 \%$ menor al del bosque nativo, es importante notar que esto se da en una superficie cercana al tercio de la superficie de bosque nativo que se contabiliza en 
el inventario GEl. Las emisiones de las plantaciones como cosecha y consumo de leña son nueve veces mayor a las emisiones del bosque nativo contabilizado. Lo anterior debido principalmente a la baja utilización de los bosques nativos respecto de los bosques plantados. Por otra parte, las plantaciones contribuyen con casi la totalidad del aporte de absorciones de carbono de los productos de madera.

En el contexto actual, donde se busca aumentar la participación de materias primas sustentables y renovables, tanto en la producción de energía como en el ámbito productivo, la biomasa y la madera jugarán un rol más preponderante cada día, lo que influirá en su rol como sumidero de carbono. Esto se conecta con los compromisos país en el marco del Acuerdo de Paris, donde hay un compromiso de generar nuevas plantaciones, incluyendo plantaciones permanentes de especies nativas y aumentando la superficie de bosque nativo manejado.

Las actuales tasas de crecimiento del bosque nativo, y por tanto sus tasas de captura de carbono, pueden aumentarse notoriamente mediante manejo silvícola sustentable efectuado en forma oportuna. Esto aumentaría la participación de los productos aserrables, con el consecuente aumento de carbono capturado en los productos de madera recolectada y desplazando emisiones de otros materiales de construcción. Lo que se conjuga, además, favorablemente, con el incentivo a la construcción con distintos productos en base a madera.

Finalmente, no solo la mejora en las tasas de absorción, sino también la disminución de las tasas de emisión redundará en mayores capturas netas. En este aspecto, la diversificación de las especies forestales utilizadas en los diversos compromisos internacionales y el aumento de la complejidad estructural de los bosques nativos promoverá una mejor forma de enfrentar la amenaza de los incendios forestales.

\section{REFERENCIAS}

Büchner, C., Martin, M., Sagardia, R., Avila, A., Molina, E., Rojas, Y., Muñoz, J. et al. (2018). Disponibilidad de Madera de Plantaciones de Pinus radiata, Eucalyptus globulus y Eucalyptus nitens 2017 - 2047. Instituto Forestal, Chile. Informe Técnico N²20. 123 p. https://doi.org/10.52904/20.500.12220/28294

CONAF. (2021). Incendios Forestales. Estadísticas Históricas. En: https://www.conaf.cl/incendiosforestales/incendios-forestales-en-chile/estadisticas-historicas/ Consulta: 27 octubre, 2021.

Donoso P., Donoso, C. \& Sandoval, V. (1993). Estudio de crecimiento de plantaciones y renovales manejados de especies nativas en el área andina de las provincias de Cautín y Valdivia. Ciencia \& Investigación Forestal, 7(2): 255-287. https://doi.org/10.52904/0718-4646.1993.188

Echeverría, C., Coomes, D., Salas J., Rey-Benayas JM., Lara A. \& Newton A. (2006). Rapid deforestation and fragmentation of Chilean temperate forests. Biol Conserv., 130:481-494. https://doi.org/10.1016/j.biocon.2006.01.017

Gobierno de Chile. (2020). Contribución determinada a nivel nacional (NDC) de Chile, Actualización 2020. 51 p. https://mma.gob.cl/wp-content/uploads/2020/04/NDC_Chile_2020_espan\%CC\%83ol-1.pdf

González, M.E., Sapiains, R., Gómez-González, S., Garreaud, R., Miranda, A., Galleguillos, M., Jacques, M. et al. (2020). Incendios forestales en Chile: causas, impactos y resiliencia. Centro de Ciencia del Clima y la Resiliencia $(\mathrm{CR})^{2}$, Universidad de Chile, Universidad de Concepción y Universidad Austral de Chile.

Grosse, H. (Ed). (2009). Silvicultura del bosque nativo chileno. Instituto Forestal. Santiago. 136 p.

INFOR. (2010). Anuario Forestal 2010. Boletín Estadístico № 128. Instituto Forestal, Chile.143 p.

INFOR. (2011). Anuario Forestal 2011. Boletín Estadístico № 132. Instituto Forestal, Chile.146 p.

INFOR. (2016) Los Recursos Forestales en Chile. Informe Final, Inventario Continuo de Bosque nativo y Actualización de Plantaciones Forestales

INFOR. (2018). Anuario Forestal 2018. Boletín Estadístico № 163. Instituto Forestal, Chile.198 p.

INFOR. (2019). Anuario Forestal 2019. Boletín Estadístico № 168. Instituto Forestal, Chile. 208 p.

INFOR. (2020 a). Anuario Forestal 2020. Boletín Estadístico № 174. Instituto Forestal, Chile. 256 p. 
INFOR. (2020 b). Propuesta y Manejo de Bosque Nativo para Diferentes Alternativas de Comercialización de la Madera. Proyecto Financiado a través del Fondo FIC-R del Gobierno Regional y su Consejo Regional. BIP 30485696-0. Informe Final. Instituto Forestal, Chile. 162 p.

IPCC. (2006). Directrices del IPCC de 2006 para los inventarios nacionales de gases de efecto invernadero. Volumen 4: Agricultura, silvicultura y otros usos de la tierra.

Martin, M., Büchner, C., Sagardia, R., Bahamondez, C., Rojas, Y., Guzmán, F., Barrientos, M. et al. (2020). Disponibilidad de Madera Nativa en Renovales de Roble - Raulí - Coihue, Regiones de Nuble a Los Ríos. Informe Técnico $\mathrm{N}^{\circ}$ 237. Instituto Forestal, Chile. 78 p.

MMA (Ministerio del Medio Ambiente). (2021). Informe del Inventario Nacional de Chile 2020: Inventario nacional de gases de efecto invernadero y otros contaminantes climáticos 1990-2018. Oficina de Cambio Climático. Santiago, Chile.

Müller-Using, S. (Ed). (2020). El manejo de Renovales en una resumida mirada: estadísticas e investigaciones en curso. Documento de Divulgación 52. Instituto Forestal, Chile. 60 p.

Pilquinao, B., Martin, M., Müller-Using, S., Rojas, Y., Villalobos, E., Guíñez, R., \& Barrientos, M. (2020). 30 años de manejo de renovales de roble: un análisis de la reacción en crecimiento y estructura. Ciencia \& Investigación Forestal, 26(3): 7-32. https://doi.org/10.52904/0718-4646.2020.535

SIMEF. (2020). Reporte Consumo de Leña por Región y Comuna. En: https://simef.minagri.gob.cl/herramientas/reporte-estadistico-ver 